\title{
BMJ Open Qualitative exploration of the intersection between social influences and cultural norms in relation to the development of alcohol use behaviour during adolescence
}

\author{
Georgie J MacArthur (D), Matthew Hickman (D), Rona Campbell
}

To cite: MacArthur GJ, Hickman M, Campbell R. Qualitative exploration of the intersection between social influences and cultural norms in relation to the development of alcohol use behaviour during adolescence. BMJ Open 2020;10:e030556. doi:10.1136/ bmjopen-2019-030556

- Prepublication history and additional material for this paper are available online. To view these files, please visit the journal online (http://dx.doi. org/10.1136/bmjopen-2019030556).

Received 19 March 2019 Revised 15 January 2020 Accepted 04 February 2020

Check for updates

(c) Author(s) (or their employer(s)) 2020. Re-use permitted under CC BY. Published by BMJ.

Department of Population Health Sciences, Bristol Medical School, University of Bristol, Bristol, UK

\section{Correspondence to} Dr Georgie J MacArthur; georgie.macarthur@bristol. ac.uk

\section{ABSTRACT}

Objectives Few contemporary studies have examined peer and social drivers of alcohol use during midadolescence. We sought to explore young people's perspectives on socio-cultural influences relating to alcohol use behaviour during this period.

Design Qualitative research study.

Methods Semi-structured one-to-one $(n=25)$, paired $(n=4)$ or triad $(n=1)$ interviews and one focus group $(n=6)$ were conducted with 30 young people aged 14 to 15 (13 males, 17 females) recruited from 4 schools, and 12 participants (aged 14 to 18, 8 males, 4 females) recruited from two youth groups in an urban centre in the West of England. Nineteen participants abstained from alcohol use, 9 were occasional or moderate drinkers and 14 drank alcohol more regularly. Interviews were audio-recorded, transcribed verbatim and analysed thematically using NVivo V.10, through a lens of social influence and social norms theories.

Results Alcohol consumption was associated with being cool, mature and popular, while enabling escape from reality and boosting confidence and enjoyment. Positive expectancies, alongside opportunity, contributed to motivating initiation, but social influences were paramount, with participants describing a need to 'fit in' with friends to avoid social exclusion. Such influences positioned drinking at parties as a normative social practice, providing opportunities for social learning and the strengthening of peer norms. Social media presented young people with positive alcoholassociated depictions of social status, enjoyment and maturity. This intersection of influences and norms generated a pressurised environment and a sense of unease around resisting pressures, which could elicit stigmatising insults. Conclusions Cultural norms, social influences and social media intersect to create a pressurised environment around alcohol use during mid-adolescence, driving the escalation in the prevalence of excessive consumption at this stage. New interventions need to address normative influences to enable the prevention of excessive alcohol use during adolescence.

\section{INTRODUCTION}

Although downward trends in alcohol use have been reported in the USA and Europe, ${ }^{1}$
Strengths and limitations of this study

- Strengths of the study include the gender-balanced sample of young people from both schools and youth groups, including those who used alcohol and those who abstained.

- One-to-one interviews were conducted, which provided space for young people to share their views around drinking, without concerns around disagreement or judgement from peers or friends.

- Limitations of this study include the lack of triangulation of data, and participants were recruited from urban and suburban settings only, meaning that our findings cannot necessarily be generalised to different geographical or demographic contexts.

the reasons for this decline are as yet unclear, and while evidence is scant, studies indicate that the decline may not be uniform across all socio-demographic or levels of alcohol consumption. ${ }^{3}$

Despite these declines in the UK, recent figures show that $24 \%$ of 15 year olds in the UK have had a drink in the last week, ${ }^{4}$ and by the age of 17 , half of girls, and nearly twothirds of boys, report drinking every week. ${ }^{5}$ Adolescent drinking in the UK therefore remains a public health concern. Guidance from the Chief Medical Officer for England states that an alcohol-free childhood is the healthiest and best option, and if children do drink alcohol, it should not be until at least the age of 15 years; while if those aged 15 to 17 do drink, they should do so infrequently and on no more than 1 day per week. ${ }^{6}$

The critical role of both peer and parental influences in relation to adolescent alcohol consumption is well documented. Quantitative and qualitative studies have highlighted the impact of factors including parental monitoring, attitudes, communication and 
parental provision of alcohol via direct and indirect mechanisms. ${ }^{7-12}$ In addition, peer influences, peer selection, the nature and reciprocity of friendships and the social context of drinking, together generate peer group-based behavioural drivers of alcohol consumption, ${ }^{13-21}$ and both descriptive and injunctive norms have been shown to be positively associated with alcohol use. ${ }^{22}{ }^{23}$ Exposure to friends' alcohol-related content via social media has also been associated with alcohol use and later heavy episodic drinking, via the development of favourable peer injunctive norms, ${ }^{24}$ demonstrating the links between use of social media, norms and social influences.

Normative social influence describes the social and emotional motivation to comply with the majority and the expectations of others to gain social approval and/ or to avoid ridicule and social rejection, particularly in public settings where lack of conformity may be more evident. ${ }^{25} 26$ Perceptions of the consensus view in a group, or collective norms, influence conformity, since the more consensual the group, the more isolated the 'deviant' and the more powerful the group in shaping the social space. In this way, alcohol use behaviour can be influenced by perceived descriptive norms (the perceived prevalence of alcohol consumption) and injunctive norms (the perceived approval of alcohol use) among young people and both have been shown to influence adolescent alcohol use at the individual and group level. ${ }^{2728}$

While qualitative studies have reported the effect of peer, parental and wider cultural influences on young people's drinking, interaction and engagement with social networking sites (SNS) and the integral nature of the social context, ${ }^{13-15}$ 29-32 few recent qualitative studies have involved an in-depth examination of social and cultural influences on the initiation and escalation of alcohol use during early-adolescence to mid-adolescence in England, and the interaction between such behavioural influences. We sought to obtain data on social influences that would be applicable to the national context to inform development of preventive interventions. In addition, since little data are available to help to explain downward trends in alcohol use among young people in England, ${ }^{2} 333$ it also remains important to gain a contemporary understanding of the impact of peers, social influences and cultural factors to offer insights around factors that might affect such trends.

In this paper, we report findings of a qualitative study that used theories of social influences and social norms as the theoretical lens through which to explore the social, cultural and behavioural drivers of alcohol consumption and the nature of drinking culture in mid-adolescence. Our exploration of the social worlds and drinking cultures of younger adolescents, and influences on drinking practice, aims to inform the development of interventions to prevent excessive alcohol consumption and related harm.

\section{METHODS}

While the initial aim of the study was to investigate the role of friendships in relation to drinking behaviour, we report here our findings regarding this and a wider range of determinants of behaviour, which reflects additional topics pertinent to young people that were raised by them and discussed in the interviews and which thereby feature in the data analysis.

\section{Sampling and recruitment}

Our study sample included 30 young people aged 14 to 15 years who were recruited from four secondary schools in the South West of England. No participants dropped out of the study. The schools were in urban $(n=3)$ and suburban/rural $(n=1)$ areas and were in areas with diverse levels of deprivation as reflected by the index of multiple deprivation score for the ward. The index of multiple deprivation score for the ward within which the school was located was identified. Schools were grouped by Index of Multiple Deprivation (IMD) score and a random number generator used to select a school from each group to be contacted regarding participation. The final sample of four schools represented wards with varied ward-level IMD scores. Students were randomly selected from the year group and snowball sampling was employed subsequently to recruit additional participants. A further 12 participants aged 14 to 18 years were recruited from youth groups, via leaders of the youth group, to ensure diversity in the sample in relation to socio-demographic characteristics and to enable data collection outside of the school environment. Leaders of youth groups disseminated information about the study and study materials and arranged interviews or the focus group. The sample included individuals who had, and hadn't, consumed alcohol. Parental consent and participant assent were given prior to participation for those aged under 16. Participants aged over 16 years gave informed consent. Recruitment took place between January 2017 and October 2017. The number of participants recruited was determined by the point at which saturation was reached, that is, when no new themes or perspectives were emerging in the interviews.

\section{Data collection}

A total of 36 semi-structured interviews were conducted (15 males, 21 females), four of which were paired interviews, and one of which was a triad interview, conducted with friends. Interviews were conducted in meeting rooms in schools $(n=30)$ and youth groups $(n=6)$. One focus group ( $\mathrm{n}=6$ males) was also conducted in a youth group. Interviews were conducted by GJM (PhD), a female researcher with training in qualitative research. No relationship was established with study participants before commencement of data collection. Before starting the interview, participants were aware of the reasons for doing the research and the interviewer's aim of developing a preventive intervention in the future. Interviews were facilitated by a topic guide, which was used flexibly so that participants could explore views and opinions which 
were meaningful to them. The topic guide included questions around attitudes and perspectives around drinking, personal histories of alcohol use, risks and consequences of drinking, the role and influence of friendship groups and social networks and family-related factors. Views on alcohol-related education and alcohol-related interventions were discussed but will be reported elsewhere. The participant sample included those who consumed alcohol and those who abstained, but many of those who chose not to drink alcohol discussed their views around alcohol use and perceptions and experiences relating to peers who did consume alcohol. Drinking patterns of participants are noted alongside quotes, based on comments made by participants during interviews. Interviews lasted on average $39 \mathrm{~min}$ (range 19 to $66 \mathrm{~min}$ ) and were audiorecorded and transcribed verbatim. Interview data were anonymised. Participants received a $£ 15$ gift voucher for taking part.

\section{Data analysis}

Transcripts of interviews were imported into NVivo V.10 (QSR International, Brisbane) and analysed thematically using this software by GJM. Participants did not check or correct transcripts. Analysis aimed to capture emergent concepts and thus took an inductive approach. Transcripts were read and re-read and open line-by-line coding was conducted with a focus on understanding and capturing participants' experiences, behaviours, feelings, attitudes and perceptions around alcohol use. We sought to explore relationships and patterns both within and between accounts. Different accounts were compared and contrasted, such that codes were progressively refined, and connections mapped, to characterise relationships and identify underlying concepts and then categories and themes. Ongoing engagement with the data ensured that emerging concepts were grounded in participants' accounts. Throughout the process the researcher (GJM) wrote analytical memos and notes to capture thoughts around meanings, themes and relationships between themes. Emerging themes and concepts, and the theoretical basis for analysis, was discussed with the last author. Thematic patterns were considered within and across cases and between groups of cases for example, those who did, or did not, drink alcohol.

Sociological theories relating to social influences, group identity and social norms were explored during analysis to enhance understanding around the theoretical basis of influences on the initiation and maintenance of alcohol use behaviour evident from the data. ${ }^{25} 3435$ Thus, these theories were used as a theoretical lens, which helped to explain the data. We also explored whether social practice theory ${ }^{36-38}$ might help to explain alcohol use behaviour as a collective social practice during adolescence. However, while a social practice perspective helped to explain the social context of alcohol use and the meanings associated with alcohol consumption, it did not fully explain data regarding peer influences and/or pressures, which were key themes in our study. We also note that we did not specifically seek to examine the influence of social media in initial interviews, rather, the role and importance of social media emerged as a theme and was therefore explored in relation to social influences during data analysis.

\section{Public involvement}

We did not involve young people in this study directly, however, the authors engaged with young people advisory groups (YPAGs) prior to commencing the programme of research in which this qualitative study was embedded. The overall aim of the programme of research was to develop an intervention to reduce excessive alcohol use and harm among young people. Together, this qualitative study, and engagement with YPAGs, aimed to inform the design and theoretical basis of an preventive intervention.

\section{RESULTS}

The major themes that emerged from the data were: normalisation and the wider culture, motivations, peer influence, pressure among peers, social media and young people's drinking culture. Family influences were also a major theme, but we have not addressed them here, as we have previously reported similar findings elsewhere. ${ }^{10}$

\section{Normalisation of drinking and the wider alcohol culture}

Young people described alcohol consumption as a normalised practice and an accepted part of the cultural context. Young people accepted drinking as part of life and stressed the importance of autonomy around people's decisions regarding alcohol consumption. Exposure to teen films and social media presented alcohol consumption as fun and 'cool' and a means of enhancing popularity, while lacking details of negative consequences. Young people therefore frequently came into adolescence with clear preformed positive attitudes towards drinking and an understanding of its meanings in the social world (online supplementary material S1, quotes 1 and 2).

So it's just like you just grow up with like people around you like just drinking casually like with meals and stuff so no-one sees it as particularly dangerous... until like something actually goes wrong. Yeah. So you're kind of shielded from the actual dangers...

(ID 17, F, drinker)

Reflecting the assimilation of wider cultural norms, perceptions of what constituted reasonable alcohol use involved levels of consumption far above levels at which drinking would become hazardous or harmful (see also online supplementary material S1, quote 3 ).

INT: And how much do you think is ok would you say?

RES: Not getting drunk like really drunk like you can't even walk. (ID 29, M, non-drinker)

Despite such misperceptions, participants (including those who did and did not drink alcohol) were aware of 
the balance between consuming alcohol for enjoyment and negative consequences, evidencing a clear awareness of the potential for a range of negative consequences to ensue if limits were overstepped, rendering the activity pointless or creating difficulties for others (online supplementary material S1, quotes 4 and 6). In addition, those who abstained, while noting acceptance of alcohol use within social groups, highlighted avoidance of such consequences through the absence of drinking in their own world.

I kind of fear the consequences of me drinking. And I also think about my family: if they were to find out they would just be like angry and annoyed at me. (ID 16 , M, non-drinker)

\section{Motivations to drink and the initiation of alcohol use}

Drinking became an integral practice in young people's social worlds by age 14 to 16 . Young people most frequently described a shared meaning of alcohol consumption relating to collective fun, enjoyment and being cool. Drinking also improved confidence, popularity and engagement with friends while enabling young people to rebel, make memories and experiment, while providing a means of managing mental illness, avoiding reality, excusing behaviour or escaping stress (online supplementary material S2, quotes 1 and 2).

Satisfying the range of motivations to drink was straightforward since alcohol was both readily available and cheap. Some described initiation of drinking among peer groups via an influential individual, who was the first to provide or drink alcohol (often via parents), setting an expectation of drinking at subsequent parties.

RES: I think first party was in October when it was like, oh there's going to be alcohol there. ...And that was sort of an initiation. (LAUGHS) I don't know.

INT: Yeah.

RES: But it's sort of like the - yes it's after that party then it's sort of expected that there is at other ones, maybe. (ID 8, F, non-drinker)

\section{Young people's drinking and party culture}

Drinking practice was rooted in house parties, which were viewed as a safer and more controlled setting than were outdoor settings. Parties' meanings were centred around the inherently social nature of the events, providing opportunities for socialising, shared experiences and a sense of togetherness with friends, as well as opportunity for drunkenness, which was a widely accepted norm. For many, alcohol made parties more enjoyable (online supplementary material S3, quote 1) and getting drunk provided an aim and purpose, while providing the means of looking popular, 'hard' or cool. Thus, widely held understandings, or aims, were to drink and 'get smashed' (online supplementary material S3, quotes 2 and 3), with the pace and extent of drunkenness often being a source of direct or indirect competition. Participants also described indirect pressures involving stigmatising insults linked to competence around intoxication.

RES: ...it's kind of like who can drink more and not get drunk, but it's like not a competition in that way, yeah.

\section{INT: So it's not a competition?}

RES: No, it's not a competition, but it feels like that because after you're a bit, oh, this guy's a lightweight, and that. (ID 37, M, non-drinker)

Thus, it was important to tread a line between appearing competent in tolerating alcohol to avoid stigmatising labelling as a 'lightweight' (online supplementary material S3, quote 4), but to remain in control to avoid ruining a party and/or require care from friends, which was associated with reputational risk and disgruntlement from friends. Those who often took on the role of looking after friends described feelings of reluctance and resentment around missing out on enjoyment, or concern around managing the effects of intoxication.

The first feeling is panic and then you feel just disappointed and you're just angry. You feel... I feel a little bit angry but especially if they've done it before you know what you've done, you know how like what your limit is and yet you go out of your way to do it... (ID 15 , F, moderate drinker)

The disparity between individual autonomy in decisions around intoxication and the requirement for help from others thereafter was also viewed negatively (online supplementary material S3, quote 5).

\section{Social influences on adolescent alcohol use}

Peer influence

Social influences became paramount in mid-adolescence, with young people frequently reporting a desire to 'fit in' with friends. Those who drank earlier and to a greater extent acted as influential 'role models' for drinking (online supplementary S4.1, quote 1). Thus, young people implicitly understood the social significance of joining in and facilitating belonging in the social world to enhance social status or to avoid social sanctions.

Kind of like 'cause everyone else is around you drinking, you kind of feel like you have to otherwise you don't really fit in um so that's what kind of happens to me most of the time. (ID 27, F, drinker)

Social influences also facilitated a rise in the prevalence of drinkers, thus enhancing proximal social influences and further contributing to the normalisation of alcohol consumption and the feedback cycle of conformity, with those who abstained thus feeling more isolated and dependent on non-drinking friends to diminish felt pressures.

Social influence led to perceived requirements to join in in some way, and those who didn't join in with the vibe of the party were a 'buzz kill' (online supplementary 
material S4.1, quote 2). As such, a somewhat circular effect sustained the party culture, whereby the increasing prevalence of drinking strengthened group norms and social learning processes that generated a desire to drink to feel included, and which generated a social environment in which abstaining could be alien (see also online supplementary material S4.1, quote 3).

I didn't intend to drinking alcohol last year... but it just kind of happens, like when you're invited to a party you just go and there's alcohol and you get, you get told there's going to be alcohol, and you just drink it to have a good time anyway 'cause everyone else is. (ID 39, M, previous, now infrequent drinker)

Those who didn't comply with norms described feelings of awkwardness, embarrassment or boredom or they avoided parties altogether, while others were incentivised to drink to avoid having to look after intoxicated friends (online supplementary material S4.1, quotes 4-6).

Notably, however, peer influences could have countereffects, such that participants described alcohol consumption as pointless, expressing confusion around friends' drinking practices, while others reported aversion to role models of intoxication, which could strengthen views around abstinence (see also online supplementary material S4.1, quote 7).

I wouldn't do it because I have seen what in the middle, I have seen people feeling sick, all in the bath, like going on the street, I was like no way I am not doing that, you see what the impacts are if you drink. You see what would happen and I don't want that at all. (ID 6, F, non-drinker)

Such individuals were less compliant, or non-compliant, with the group norm and often played a greater 'mum' role in supporting and helping others within the social group (online supplementary material S4.1, quote 8). Those who were non-compliant, acted in keeping with family values and messages, and were unaffected by group norms.

I don't know what, what my mum would think if I become a drinker, so I - and I think she's kind of built it in me that it's, it's now in my moral code not to drink.

(ID 12, M, non-drinker)

Individuals who abstained from drinking described the importance of friends with similar attitudes and behaviours around drinking and there was some evidence for peer selection in this group. Such friends provided support for their viewpoint and provided some protection from felt pressure or exclusion, enabling them to abstain and/or to enjoy social events more.

...if I was on my own it would have been worse, because you're just looking around and everyone's drinking. Not that it would lead me to drinking, but it's still better that I had friends who just don't do that at all: it made it much more fun. (ID 16, M, non-drinker)

Those who abstained frequently described strong interests in extracurricular activities, particularly sports, a strong religious faith and/or clear core values and aligned family viewpoints (which highlighted strong, clearly stated disapproval for alcohol use), which altered the meaning of alcohol use and reduced, or counteracted, the effects of social influences and norms (online supplementary material S4.1, quote 9 ). In addition, some of those who abstained reported family history of awareness of dependence or alcohol-related problems, which increased concern around the risks and negative consequences of alcohol consumption.

I'm pretty sure I'm glad not to like I don't want to start doing it because then maybe like later life I will be addicted and I don't want to be. (ID20, M, non-drinker)

\section{The influence of social media}

The use of social media was integral to adolescent drinking behaviour and practice, particularly use of Instagram or the multimedia messaging app Snapchat. Posting pictures and videos from parties reflected the meanings associated with alcohol use, thus portraying participants being cool and sociable, to enhance popularity and social status. The ubiquity and prominence of social media weaved into young people's lives a relatively consistent, positive view of alcohol consumption that could act as another source of social influence and a visual depiction of norms that could contribute to a felt pressure to drink (see also online supplementary material S4.2, quotes 1 and 2).

Because in a friendship it's more like direct, like they're going to tell you that, but like, on a big scale like social media it kind of like tells you like that you should be drinking, like when you can, and the norm is to, and then I think you feel a bit pressured into it. (ID 37, M, non-drinker)

Social media used to demonstrate social capital, drinking 'competence' and maturity. Some were described as sipping drinks just to look right in pictures, while others aimed to depict their prowess in consumption.

I've noticed that a lot in my year group and like they'll kind of just... even if they don't really want to drink it they'll kind of just hold it there and they'll just like sip and stuff so they can have it for photos. (ID 34, F, moderate drinker)

Nevertheless, views on the impact on social media were mixed. A minority expressed caution about posting images of themselves drinking owing to potential reputational risk. A minority also reported a view that drinking was unaffected by social media, although it was nevertheless described as a key component of drinking practice. 


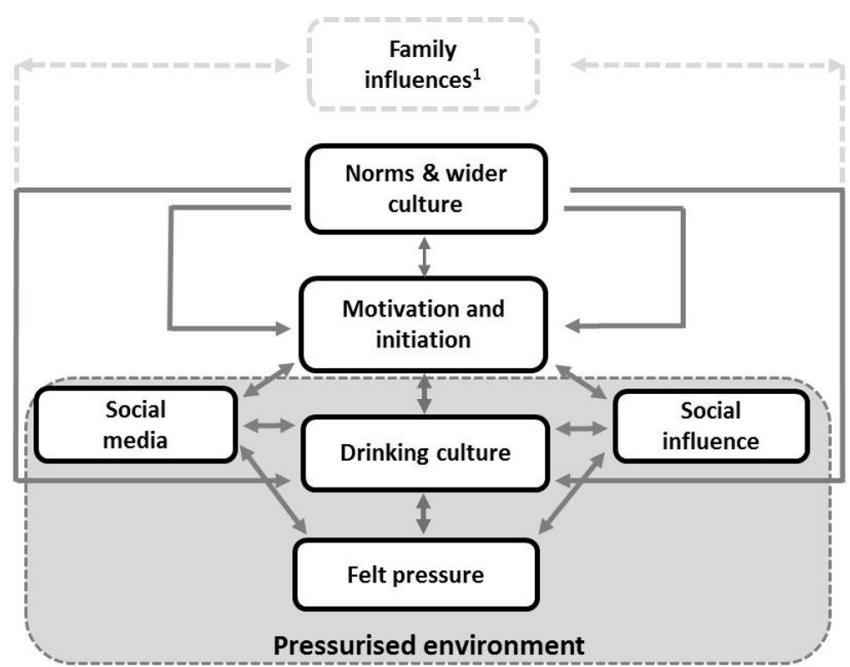

Figure 1 Intersection of social and cultural influences on adolescent alcohol use and generation of a pressurised environment. ${ }^{1}$ Family influences have been addressed elsewhere.

\section{The pressurised environment}

The combination of social media, social influences and social learning, alongside the meanings associated with drinking (eg, belonging, popularity) and wider cultural influences, created a pressurised environment around drinking (figure 1). Thus, the very interest in fitting in or feeling a need to join in with friends was felt to be subtly pressurising, while being the only person not drinking felt awkward and created unease, further contributing to a felt pressure.

Because you see everyone's doing it then you felt... er like obliged to do it as well sometimes. Um I don't often, like, myself but you can definitely tell other people um it's like, oh everybody else is doing it so then I have to do it as well to fit in, it's like one of those sorts of things and also if your friends are drunk then it sort of becomes a thing where oh do you want mine you can have some, have some, and then you're like oh my gosh like do I accept, do I not. (ID 15, F, moderate drinker)

Branding of individuals as 'wimps' or awarding 'lad points' for intoxication associated with courage and humour, further created incentives and obligations to drink to excess (online supplementary material S5, quote 1). The inextricable link between drinking alcohol and socialising meant that resisting pressures or challenging friends risked losing credibility as an integral and fun group member, or risked jeopardising friendships (see also online supplementary material S5, quotes 2 and 3).

INT: ... and how does that make you feel if you are saying no?

RES: Um if you're like receiving yeah then you can kind of feel like it makes you not want to be in that situation, it makes you just like feel oh I don't feel safe in this environment anymore cos now I'm doing something that I don't want to do and you're just like what do I do, I can't deal with this situation and you don't want to not be friends with people who you're friends with, you don't want to break friendships over it but it's one of those things where you kind of if you don't like it then you have to say no and if your friends didn't accept that then it's like you have to sort that out with your friends yourself, that sort of thing. (ID 15, F, moderate drinker)

Pressure to drink was felt in a range of ways, including subtle and indirect pressure through offers of alcoholic drinks, the felt need to be fun and to fit in, or proximal social influence. However, participants also reported more direct pressure through goading or direct insults, leading some to describe feeling 'forced to drink' and others wishing to avoid appearing cowardly. Overall, the social environment was felt to be conducive to feeling obliged to drink, or being 'pushed in the direction' towards alcohol use (see also online supplementary material S5 quotes 4 and 5).

... I used to think alcohol was really bad but I guess as like it becomes more talked about, you're kind of dragged into it.

INT: Mm. It's interesting you say dragged into it.

RES: Not dragged - I'd say like kind of um pushed in the direction. (ID 27, F, drinker)

Nevertheless, such felt pressures were often unclear to the friends in question who were drinking, who therefore pressurised others unknowingly. Drinkers did not consider their actions to be pressurising, rather a gesture to enable or encourage friends to join in (see also online supplementary material S5, quote 6).

They just think they're saying it does - it's just a laugh like, if they're - they're kind of - they're almost trying to make their experience more enjoyable by saying if you do this... it will make it better, but I don't think that's the same kind of response for the person on the other end. (ID 33, M, drinker)

As outlined above, for non-drinkers, having friends with a similar viewpoint provided support and reduced felt pressure.

... I don't know if I didn't have my friends or anyone to tell me not to drink, I'd probably be drinking by now. ...So, I'm kind of happy that they're around. (ID 12, M, non-drinker)

\section{DISCUSSION}

In this paper, we have demonstrated how the wider alcohol culture normalised alcohol use for young people, helping to generate perceptions of drinking as a social practice associated with being cool, mature and popular as well as an important means of enhancing enjoyment and social status, in support of evidence regarding primary social 
and enhancement drinking motives in young adults. ${ }^{39}$ The ubiquity of alcohol use generated normative social influences and opportunities for social learning around initiation and continuation of alcohol use. Together with embedded meanings and expectancies, the interaction between peer norms, social influences and the effects of social media generated a pressurised environment in which young people felt obliged to comply and join in.

The strengths of our study include the gender-balanced sample of young people from both schools and youth groups, including those who used alcohol and those who abstained. We also used one-to-one interviews, which provided space for young people to share their views around drinking, without concerns around disagreement or judgement from peers or friends. A small proportion of interviews were conducted with dyads with one triad, however, we did not find evidence that participants exaggerated or withheld views and perspectives in these pairs, and paired interviews were conducted when expressed as a preference by the participants. Nevertheless, our data were not triangulated, and participants were recruited from urban and suburban settings only, so our findings cannot necessarily be generalised to different geographical or demographic contexts. Interestingly, although parental messages and the off-putting effect of observing intoxicated role models were described by some moderate drinkers, our data did not provide evidence regarding factors that might be affecting downward trends in the prevalence of alcohol use among young people in England, and further qualitative and quantitative research will be needed to explore such trends. Lastly, while we have reported that social influences and pressures are a critical influence on young people's alcohol use, further research will be needed to explore exactly how such influences could be effectively addressed in public health interventions for young people.

The frequently reported desire to fit in with the group, and unease expressed in relation to abstinence, highlighted the central role of normative social influence in generating a collective practice in which intoxication was accepted and expected. Evidence suggests that both direct and indirect pathways of peer influences and pressures may exist, which can contribute to generating social influences (and can act together with peer selection ${ }^{19} 40$ ). Pathways can be direct, including offers of drinks, dares or goading, (fitting with social and enhancement motives for drinking) ${ }^{41}$ and indirect, including modelling of peer drinking and/or contributions to the formation of norms, beliefs and expectancies, ${ }^{42}{ }^{43}$ (fitting with conformity motives for drinking) ${ }^{39}$ which were also evident in our study. In addition, studies have reported an impact of being around peers who drink alcohol, either through proximity to social acquaintances who consume alcohol or through time spent with drinking peers, ${ }^{44} 45$ highlighting the important impact of the rise in prevalence of alcohol use through adolescence. This was evident in our study since being around other drinkers and being immersed in a practice in which drinking was highly prevalent (contributing to descriptive norms) contributed to the felt obligation to drink.

Frequent reports by young people of needing to 'fit in' suggest that injunctive norms, or perceptions of peer approval of drinking, were also critical. Thus, our findings support those of quantitative studies, which have reported associations between both descriptive and injunctive norms and frequency and quantity of adolescence alcohol use and heavy episodic drinking. ${ }^{22}{ }^{46}$ Specifically, one study reported a greater impact of injunctive compared with descriptive norms, with the former associated with a range of measures of alcohol use as well as alcohol-related negative consequences. ${ }^{22}$

In addition to social norms and influences among peers, social media or SNS, played a role in providing a physical depiction of positive alcohol-related descriptive and injunctive norms, which was an integral part of the social practice of adolescent alcohol use. Thus, we have reported evidence to support a previous study describing 'intoxigenic digital identities' and 'intoxigenic digital spaces' that contribute to the normalisation of alcohol use. ${ }^{47}$ Similarly, a qualitative study of young women's drinking cultures described the critical nature of SNS in facilitating displays of popularity, belonging, membership of the social network and 'friendship fun'. ${ }^{32}$ Such media also bring about opportunities for misperceptions owing to the circulation of positive imagery, which can be interpreted at face value or as 'truth'. ${ }^{48} 49$ Quantitative data have demonstrated an association between exposure to friends' alcohol-related SNS content and initiation of alcohol use and stronger pro-alcohol peer injunctive norms, highlighting how the provision of opportunities for observation, comparison and role-modelling of behaviours through SNS can impact on norm perception and behaviour. ${ }^{24}$ In addition, others have reported an association between alcohol promotion through digital media and adolescent alcohol consumption..$^{50}$

Taken together, therefore, evidence suggests the need for public health interventions to effectively address both peer norms and social influences during adolescence, including the effects of SNS, to enhance the likelihood of effective prevention. Furthermore, the evidence of felt pressure, resulting from the influences discussed above, suggests that many young people feel a lack of autonomy around alcohol consumption, and constraint around behavioural decisions within their social groups. Thus, in addition to addressing norm perception and normative social influence, there is a need for interventions to find ways of overcoming such constraints and fostering greater resistance to conformity. Indeed our sample included participants who reported abstaining from alcohol use, and who navigated the social world resisting influences of drinking peers, with critical support from non-drinking friends, the importance of which has been reported by others. ${ }^{52}$ Thus, fostering greater resistance to conformity may require a strengthening of the voice and influence of those in the minority group (ie, those who abstain from drinking) as outlined in Moscovici's theory of minority 
influence ${ }^{53} 54$ which may be increasingly feasible in light of reported downward trends in adolescent alcohol consumption $^{3}$ and thus an increase in the proportion of non-drinking peers. Moscovici also highlights how influence goes beyond shaping people to a system, but that it continually changes a social system, thus the influence of increasing proportions of abstainers, who in our study reported counter-effects of peer influence and aversion to role models of intoxication, may increasingly shift alcohol use downwards at a population $\operatorname{level}^{25}$ as the benefits of the 'deviant' or non-conforming behaviour or perspective become evident. It has also been suggested that feelings of powerlessness during adolescence may be overcome through enhanced social status for young people, for instance through opportunities for creative activities and active engagement in communities. ${ }^{55}$

\section{CONCLUSIONS}

Our study has demonstrated the effect of the interplay between cultural norms, social influences, social pressures and social media, particularly the critical role of normative social influence, in driving the escalation and normalisation of alcohol use in mid-adolescence. Our findings suggest that there is a need for a combination of approaches to effectively prevent excessive alcohol use among young people. Interventions in young people need to target normative social influence and peer norms to interrupt the rise in prevalence of excessive drinking, while aiming to enhance social status and the ability to challenge social pressures, to increase the likelihood of preventing alcohol-related harm.

Contributors GJM was responsible for recruitment, acquisition, analysis and interpretation of data and wrote the first draft of the manuscript. MH contributed to interpretation of data and finalisation of the manuscript. RC was responsible for conceptualisation of the study and contributed to interpretation of data and finalisation of the manuscript.

Funding GJM was supported by an NIHR post-doctoral fellowship award (PDF2013-06-026). This report is independent research supported by the National Institute for Health Research (Post-Doctoral Fellowship, PDF-2013-06-026). The work was undertaken with the support of The Centre for the Development and Evaluation of Complex Interventions for Public Health Improvement (DECIPHer), a UKCRC Public Health Research Centre of Excellence. Joint funding (MR/ K0232331/1) from the British Heart Foundation, Cancer Research UK, Economic and Social Research Council, Medical Research Council, the Welsh Government and the Wellcome Trust, under the auspices of the UK Clinical Research Collaboration, is gratefully acknowledged. RC is supported by the University of Bristol.

Disclaimer The views expressed in this publication are those of the author(s) and not necessarily those of the NHS, the National Institute for Health Research or the Department of Health and Social Care.

Competing interests $\mathrm{RC}$ is a scientific advisor to Evidence to Impact a notfor-profit company wholly owned by the Universities of Cardiff and Bristol which licences, quality-assures and supports the delivery of evidence-based public health promotion interventions. $\mathrm{RC}$ receives payment for this work. $\mathrm{MH}$ has received payment for unrelated activity from Gilead Sciences Inc, Bristol Myers-Squibb and Jannsen, UK.

Patient consent for publication Not required.

Ethics approval Ethical approval for the study was obtained from the University of Bristol Faculty of Health Sciences Ethics Committee (study 131443 (8201)/2306).

Provenance and peer review Not commissioned; externally peer reviewed.
Data availability statement № data are available.

Open access This is an open access article distributed in accordance with the Creative Commons Attribution 4.0 Unported (CC BY 4.0) license, which permits others to copy, redistribute, remix, transform and build upon this work for any purpose, provided the original work is properly cited, a link to the licence is given, and indication of whether changes were made. See: https://creativecommons.org/ licenses/by/4.0/.

\section{ORCID iDs}

Georgie J MacArthur http://orcid.org/0000-0003-2047-6519

Matthew Hickman http://orcid.org/0000-0001-9864-459X

\section{REFERENCES}

1 Looze Mde, Raaijmakers Q, Bogt TT, et al. Decreases in adolescent Weekly alcohol use in Europe and North America: evidence from 28 countries from 2002 to 2010. Eur J Public Health 2015;25 Suppl 2:69-72.

2 Kraus L, Seitz N-N, Piontek D, et al. 'Are the times A-Changin'? trends in adolescent substance use in Europe. Addiction 2018;113:1317-32

3 Pape H, Rossow I, Brunborg GS. Adolescents drink less: how, who and why? A review of the recent research literature. Drug Alcohol Rev 2018;37 Suppl 1:S98-114.

4 NHS Digital. Smoking, drinking and drug use among young people. England 2016. UK: National Statistics, 2017

5 Public Health England. Data intelligence summary: alcohol consumption and harm among under 18 year olds. Contract No: publications gateway number: 2016173. UK: Public Health England, 2016.

6 Department of Health. Guidance on the consumption of alcohol by children and young people. Report from Sir Liam Donaldson, chief medical officer for England. London: Department of Health, 2009.

7 Rossow I, Keating P, Felix L, et al. Does parental drinking influence children's drinking? A systematic review of prospective cohort studies. Addiction 2016;111:204-17.

8 Mattick RP, Clare PJ, Aiken A, et al. Association of parental supply of alcohol with adolescent drinking, alcohol-related harms, and alcohol use disorder symptoms: a prospective cohort study. Lancet Public Health 2018;3:e64-71.

9 Özdemir M, Koutakis N. Does promoting parents' negative attitudes to underage drinking reduce adolescents' drinking? the mediating process and moderators of the effects of the Örebro prevention programme. Addiction 2016;111:263-71.

10 Jacob N, MacArthur GJ, Hickman M, et al. A qualitative investigation of the role of the family in structuring young people's alcohol use. Eur $J$ Public Health 2016;26:102-10.

11 Campbell JM, Oei TP. A cognitive model for the intergenerational transference of alcohol use behavior. Addict Behav 2010;35:73-83.

12 Mahedy L, MacArthur GJ, Hammerton G, et al. The effect of parental drinking on alcohol use in young adults: the mediating role of parental monitoring and peer deviance. Addiction 2018;113:2041-50.

13 MacArthur GJ, Jacob N, Pound P, et al. Among friends: a qualitative exploration of the role of peers in young people's alcohol use using Bourdieu's concepts of habitus, field and capital. Sociol Health IIIn 2017;39:30-46.

14 de Visser RO, Wheeler Z, Abraham C, et al. 'Drinking is our modern way of bonding': young people's beliefs about interventions to encourage moderate drinking. Psychol Health 2013;28:1460-80.

15 Niland P, Lyons AC, Goodwin I, et al. "Everyone can loosen up and get a bit of a buzz on": young adults, alcohol and friendship practices. Int J Drug Policy 2013;24:530-7.

16 Kelly AB, Chan GCK, Toumbourou JW, et al. Very young adolescents and alcohol: evidence of a unique susceptibility to peer alcohol use. Addict Behav 2012;37:414-9.

17 Ali MM, Dwyer DS. Social network effects in alcohol consumption among adolescents. Addict Behav 2010;35:337-42.

18 Percy A, Wilson J, McCartan C, et al. Teenage drinking cultures. York: Joseph Rowntree Foundation, 2011.

19 Leung RK, Toumbourou JW, Hemphill SA. The effect of peer influence and selection processes on adolescent alcohol use: a systematic review of longitudinal studies. Health Psychol Rev 2014;8:426-57.

20 Fujimoto K, Valente TW. Decomposing the components of friendship and friends' influence on adolescent drinking and smoking. $J$ Adolesc Health 2012;51:136-43.

21 Bot SM, Engels RCME, Knibbe RA, et al. Friend's drinking behaviour and adolescent alcohol consumption: the Moderating role of friendship characteristics. Addict Behav 2005;30:929-47. 
22 Pedersen ER, Osilla KC, Miles JNV, et al. The role of perceived injunctive alcohol norms in adolescent drinking behavior. Addict Behav 2017;67:1-7.

23 Teunissen HA, Kuntsche E, Scholte RHJ, et al. Friends' drinking norms and male adolescents' alcohol consumption: the Moderating role of performance-based peer influence susceptibility. J Adolesc 2016;53:45-54.

24 Nesi J, Rothenberg WA, Hussong AM, et al. Friends' alcohol-related social networking site activity predicts Escalations in adolescent drinking: mediation by peer norms. J Adolesc Health 2017;60:641-7.

25 Turner JC. Social influence. Buckingham: Open University Press, 1991.

26 Asch SE. Studies of independence and conformity: I. A minority of one against a unanimous majority. Psychol Monogr 1956;70:1-70.

27 François A, Johnson SL, Waasdorp TE, et al. Associations between adolescents' perceptions of alcohol norms and alcohol behaviors: incorporating Within-School variability. Am J Health Educ 2017;48:80-9.

28 Amialchuk A, Ajilore O, Egan K. The influence of misperceptions about social norms on substance use among school-aged adolescents. Health Econ 2019;28:736-47.

29 Townshend TG. Youth, alcohol and place-based leisure behaviours: a study of two locations in England. Soc Sci Med 2013;91:153-61.

30 Bremner P, Burnett J, Nunney F. Young people alcohol and influences. A study of young people and their relationship with alcohol. York, 2011.

31 Scott S, Shucksmith J, Baker R, et al. 'Hidden Habitus': A Qualitative Study of Socio-Ecological Influences on Drinking Practices and Social Identity in Mid-Adolescence. Int J Environ Res Public Health 2017; $14: 611$

32 Atkinson AM, Sumnall HR. 'If I don't look good, it just doesn't go up': A qualitative study of young women's drinking cultures and practices on Social Network Sites. Int J Drug Policy 2016;38:50-62.

33 Larm P, Livingston M, Svensson J, et al. The increased trend of non-drinking in adolescence: the role of parental monitoring and attitudes toward offspring drinking. Drug Alcohol Rev 2018;37(Suppl 1):S34-41.

34 Tajfel H, Flament C, Billig MG, et al. Social categorisation and intergroup behaviour. Euro J of Soc Psychol 1971;5:245-53.

35 Sherif M. The psychology of social norms. New York: Harper and Brothers, 1936

36 Blue S, Shove E, Carmona C, et al. Theories of practice and public health: understanding (un)healthy practices. Crit Public Health 2016;26:36-50.

37 Shove EPM, Watson M. The dynamics of social practice: everyday life and how it changes. London: Sage, 2012.

38 Meier PS, Warde A, Holmes J. All drinking is not equal: how a socia practice theory lens could enhance public health research on alcohol and other health behaviours. Addiction 2018;113:206-13.
39 Mackinnon SP, Couture M-E, Cooper ML, et al. Cross-Cultural comparisons of drinking motives in 10 countries: data from the DRINC project. Drug Alcohol Rev 2017;36:721-30.

40 Osgood DW, Ragan DT, Wallace L, et al. Peers and the emergence of alcohol use: influence and selection processes in adolescent Friendship networks. J Res Adolesc 2013;23:500-12.

41 Kuntsche E, Knibbe R, Gmel G, et al. Why do young people drink? A review of drinking motives. Clin Psychol Rev 2005;25:841-61.

42 Borsari B, Carey KB. Peer influences on College drinking: a review of the research. J Subst Abuse 2001;13:391-424.

43 Studer J, Baggio S, Deline S, et al. Peer pressure and alcohol use in young men: a mediation analysis of drinking motives. Int J Drug Policy 2014;25:700-8.

44 Dallas R, Field M, Jones A, et al. Influenced but unaware: social influence on alcohol drinking among social acquaintances. Alcohol Clin Exp Res 2014;38:1448-53.

45 Salvy S-J, Pedersen ER, Miles JNV, et al. Proximal and distal social influence on alcohol consumption and marijuana use among middle school adolescents. Drug Alcohol Depend 2014;144:93-101.

46 Song E-Y, Smiler AP, Wagoner KG, et al. Everyone says it's OK: adolescents' perceptions of peer, parent, and community alcohol norms, alcohol consumption, and alcohol-related consequences. Subst Use Misuse 2012;47:86-98.

47 Griffiths R, Casswell S. Intoxigenic digital spaces? Youth, social networking sites and alcohol marketing. Drug Alcohol Rev 2010;29:525-30.

48 Moreno MA, Briner LR, Williams A, et al. A content analysis of displayed alcohol references on a social networking web site. $J$ Adolesc Health 2010;47:168-75.

49 McCreanor T, Lyons A, Griffin C, et al. Youth drinking cultures, social networking and alcohol marketing: implications for public health. Crit Public Health 2013;23:110-20.

50 Lobstein T, Landon J, Thornton N, et al. The commercial use of digital media to market alcohol products: a narrative review. Addiction 2017;112(Suppl 1):21-7.

51 Scott S, Muirhead C, Shucksmith J, et al. Does Industry-Driven alcohol marketing influence adolescent drinking behaviour? A systematic review. Alcohol Alcohol 2017;52:84-94.

52 Rees C, Wallace D. The myth of conformity: adolescents and abstention from unhealthy drinking behaviors. Soc Sci Med 2014;108:34-45.

53 Moscovici S, Personnaz B. Studies in social influence. V. minority influence and conversion behavior in a perceptual tast. $J$ Exp Soc Psychol 1980;16:270-82.

54 Moscovici S, Lage E. Studies in social influence III: majority versus minority influence in a group. Eur J Soc Psychol 1976;6:149-74.

55 Pound P, Campbell R. Locating and applying sociological theories of risk-taking to develop public health interventions for adolescents. Health Sociol Rev 2015;24:64-80. 